\title{
A Case Study on A Miner Dataset: Identifying leading research through various Models
}

\author{
Dr.G.Ayyappan $^{1}$, Dr.C.Nalini $^{2}$, Dr.A.Kumaravel ${ }^{3}$ \\ Associate Professor, Department of Computer Science, Bharath University ${ }^{1}$ \\ Professor, Department of Computer Science, Bharath University ${ }^{2}$ \\ Professor \& Head, School of Computing, Bharath Unviersity ${ }^{3}$ \\ ayyappangmca@gmail.com ${ }^{1}$,drnalinichidambaram@gmail.com², drkumaravel@gmail.com ${ }^{3}$
}

\begin{abstract}
The increasing tendency across scientific disciplines to write multi authored papers [1,2] makes the issue of the sequence of contributors' names a major topic both in terms of reflecting actual contributions and in a posteriori assessments by evaluation committees. The reviewers aware that there are different cultures to authorship order. The usual and informal practice of giving the whole credit (impact factor) to each author of a multi authored paper is not adequate and over emphasizes the minor contributions of many authors. Similarly, evaluation of authors according to citation frequencies means often overrating resulting from high-impact but multi authored publications. Teja Tscharntke et al. [72] proposed that four methods. Like as SDC,EC, FLAE, and PCI. Comparison of the credit for contributions to this study under the four different models has been suggested. The proposed systems, such as Individual Frequency (IF) and Weighted Frequency (WF), have no repeated impact for each position.
\end{abstract}

Keywords: SDC,EC,FLAE,PCI,IF and WF.

\section{INTRODUCTION}

Social network is a structure of human relations and association. It is made up of a organized social actors in a network form. Information has varied number of forms and various purposes for communication. Journals serve as major source of primary information. The Researchers tend to publish more and more research output in journals. This paper focuses on the novel techniques for identifying the leading research contribution in the clusters of research social networks. M. T. Rahman et al. proposed an approach that can be used to measure the impact of an author of a multi-authored paper in a more accurate way than either giving each author full credit or dividing credit equally. The proposed proposal not only resolved the long-standing concern for the fair distribution of each author's credit depending on his/her contribution, but it will also, hopefully, discourage the addition of non-contributing authors to a paper. Tasleem Arif proposed a method that use a token-based similarity score in this first stage of comparison and based on the results of the first stage it uses a characterbased similarity score in the second stage. Experimental results obtained on standard datasets indicated that the proposed technique shows a lot of improvements over the existing methods. J. M. Warrender proposed a simple tool that assisted researchers in assessing contributions to a scientific publication, for ease in evaluating which contributor qualify for authorship, and in what order the authors should be listed. The tool identified four phases of activity leading to a publication.

In this paper organizes section one has related works and brief introduction of these fields, In section two represents materials and methods, In section three describes results and discussions and the section four presents conclusion

\section{MATERIALS AND METHODS}

Social network analysis A "social” network is defined as a group of collaborating (or competing) entities that have some type of relationship and interact within a shared environment often referred to as a community. Author collaboration Research collaboration or Author collaboration can be defined as the working together of researchers to achieve the common goal of producing new research knowledge. The dataset named as topic_paper_author in the academic social network data from AMiner [8,9]. The dataset is collected for the purpose of cross domain recommendation. The attributes contain Data Mining, Medical Informatics, Theory, Visualization and Database areas. In this research work used Weka 3.6.9 [11], open source software for Text Mining process, MeSH [12] for identification of domain and SAS University Edition [13,14] which is getting permission to access SAS Studio from SAS Institute for Mining of the research community. Based on this novel metrics, the “Top most Influential researcher” of research community has been identified. 


\section{EXPERIMENTS AND RESULTS}

In this section presents experiment ans results of the various methods like as Sequence Credit Method, Equal Contribution (EC) Method, First Last Author Emphasis (FLAE) Method, Percent Contribution Indicated (PCI) Method, Individual Frequency Method and Weighted Frequency Method.

\section{Sequence Determines Credit (SDC) Method}

The sequence of authors should reflect the declining importance of their contribution, as suggested by the previous studies. Authorship order only reflects relative contribution, whereas evaluation committees often need quantitative measures.

Therefore, the SDC method suggested that the first author should get the credit for the whole impact (impact factor), the second author one-half of the impact factor, the third author one-third of the impact factor, and so forth, up to rank 10. When papers have more than 10 authors, the contribution of each author from the 10th position onwards was then valuated at just $5 \%$.

The leading research contributors based on the research work in the Topic_Paper_Author dataset were ranked by using the SDC method. Table 1.1 lists the top 20 leading contributors in the dataset. By using the SDC method, the leading author with the highest frequency (58.5) was identified as Mr. Surajit Chaudhuri.

Table 1.1 The top 20 authors ranking by using Sequence-Determines-Credit (SDC) Method

\begin{tabular}{|c|c|c|}
\hline S. No. & Author & Sequence-Determines-Credit (SDC) \\
\hline 1 & Surajit Chaudhuri & 58.5 \\
\hline 2 & Jiawei Han & 56.5 \\
\hline 3 & Rakesh Agrawal & 55 \\
\hline 4 & Philip S. Yu & 51.5 \\
\hline 5 & Richard T. Snodgrass & 48.5 \\
\hline 6 & NogaAlon & 46.5 \\
\hline 7 & H. V. Jagadish & 46.5 \\
\hline 8 & Hector Garcia-Molina & 42 \\
\hline 9 & Michael J. Franklin & 41.5 \\
\hline 10 & Christos Faloutsos & 41 \\
\hline 11 & Michael Stonebraker & 38.6 \\
\hline 12 & Baruch Awerbuch & 38 \\
\hline 13 & Michael J. Carey & 37.5 \\
\hline 14 & Jennifer Widom & 35 \\
\hline 15 & Piotr Indyk & 34.5 \\
\hline 16 & Jon M. Kleinberg & 34.5 \\
\hline 17 & S. Muthukrishnan & 34.5 \\
\hline 18 & Hans-Peter Kriegel & 33 \\
\hline 19 & Divesh Srivastava & 32.5 \\
\hline 20 & Kenneth A. Ross & 32.5 \\
\hline
\end{tabular}

\section{Equal Contribution (EC) Method}

Authors use alphabetical sequence to acknowledge similar contributions or to avoid disharmony in the collaborating groups. EC method suggested that the contribution of each author is valuated as an equal proportion (impact divided by the number of all authors, but a minimum of 5\%).The leading research contributors based on the research work in the Topic_Paper_Author dataset were ranked by using the EC method.

In Table 1.2 lists the top 20 leading contributors in the dataset. By using the EC method, the leading author with the highest frequency (19) was identified as Mr. MiklósAjtai. 
Table 1.2 The top 20 authors ranking by using Equal Contribution(EC) method

\begin{tabular}{|c|c|c|}
\hline S.No. & Author & Equal Contribution (EC) \\
\hline $\mathbf{1}$ & MiklósAjtai & $\mathbf{1 9}$ \\
\hline 2 & Rakesh Agrawal & 17.5 \\
\hline 3 & Matthew Andrews & 17 \\
\hline 4 & Dimitris Achlioptas & 14 \\
\hline 5 & Timothy M. Chan & 13.86 \\
\hline 6 & Shree K. Nayar & 13.26 \\
\hline 7 & Hector Garcia-Molina & 13.02 \\
\hline 8 & Jiawei Han & 13 \\
\hline 9 & Graham Cormode & 13 \\
\hline 10 & Susanne Albers & 12.4 \\
\hline 11 & Michael J. Franklin & 12.24 \\
\hline 12 & Surajit Chaudhuri & 12.21 \\
\hline 13 & Alan M. Frieze & 12 \\
\hline 14 & Michael Alekhnovich & 11.75 \\
\hline 15 & Beng Chin Ooi & 11.56 \\
\hline 16 & Christos Faloutsos & 11.55 \\
\hline 17 & David Eppstein & 11.55 \\
\hline 18 & Yannis E. Ioannidis & 11.5 \\
\hline 19 & Hongjun Lu & \\
\hline 20 & Wei Wang & 11.25 \\
\hline
\end{tabular}

\section{First Last Author Emphasis (FLAE) Method}

In many laboratories, the great importance of last authorship is well established. FLAE method suggested that the first author should get the credit of the impact factor, the last author half, and the credit of the other authors should be the impact divided by the number of all authors.

The leading research contributors based on the research work in the Topic_Paper_Author dataset were ranked by using the FLAE method.

Table 1.3 lists the top 20 leading contributors in the dataset. By using the FLAE method, the leading author with the highest frequency (49.92) was identified as Mr. Surajit Chaudhuri. 
Table 1.3 The top 20 authors ranking by using First-Last-Author-Emphasis(FLAE) method

\begin{tabular}{|c|c|c|}
\hline S.No. & Author & First-Last-Author-Emphasis (FLAE) \\
\hline 1 & Surajit Chaudhuri & 49.92 \\
\hline 2 & Rakesh Agrawal & 48 \\
\hline 3 & Richard T. Snodgrass & 40.36 \\
\hline 4 & H. V. Jagadish & 32.9 \\
\hline 5 & Jiawei Han & 30.58 \\
\hline 6 & Pankaj K. Agarwal & 30.49 \\
\hline 7 & Michael J. Franklin & 29.5 \\
\hline 8 & David Eppstein & 29.48 \\
\hline 9 & Michael J. Carey & 28.14 \\
\hline 10 & Michael Stonebraker & 28.14 \\
\hline 11 & C. Mohan & 27.32 \\
\hline 12 & Jon M. Kleinberg & 27.24 \\
\hline 13 & Avrim Blum & 27.15 \\
\hline 14 & SudiptoGuha & 26.88 \\
\hline 15 & Piotr Indyk & 26.7 \\
\hline 16 & Kenneth A. Ross & 26.26 \\
\hline 17 & David R. Karger & 25.25 \\
\hline 18 & Ling Liu & 24.52 \\
\hline 19 & Christos Faloutsos & 23.84 \\
\hline 20 & Daniel A. Keim & 23 \\
\hline
\end{tabular}

\section{Percent Contribution Indicated (PCI) Method}

There is a trend to detail each author's contribution. It has used to establish the quantified credit.

The leading research contributors based on the research work in the Topic_Paper_Author dataset were ranked by using the PCI method.

Table 1.4 lists the top 20 leading contributors in the dataset. By using the PCI method, the leading author with the highest frequency (80.5) was identified as Mr. Jiawei Han. 
Table 1.4 The top 20 authors ranking by using Percent-Contribution-Indicated (PCI) method

\begin{tabular}{|c|c|c|}
\hline S.No. & Author & Percent-Contribution-Indicated (PCI) \\
\hline $\mathbf{1}$ & Jiawei Han & $\mathbf{8 0 . 5}$ \\
\hline 2 & Philip S. Yu & 79.8 \\
\hline 3 & Surajit Chaudhuri & 68.5 \\
\hline 4 & Hector Garcia-Molina & 66.8 \\
\hline 5 & Rakesh Agrawal & 65.3 \\
\hline 6 & H. V. Jagadish & 57.3 \\
\hline 7 & Christos Faloutsos & 57.1 \\
\hline 8 & Michael J. Franklin & 55.5 \\
\hline 9 & Richard T. Snodgrass & 54.5 \\
\hline 10 & Hans-Peter Kriegel & 51.2 \\
\hline 11 & Jeffrey F. Naughton & 50.1 \\
\hline 12 & Rajeev Motwani & 49.2 \\
\hline 13 & Jennifer Widom & 48.7 \\
\hline 14 & S. Muthukrishnan & 48.4 \\
\hline 15 & NogaAlon & 46.9 \\
\hline 16 & Divesh Srivastava & 46.5 \\
\hline 17 & Arie E. Kaufman & 46.1 \\
\hline 18 & Michael Stonebraker & 45.9 \\
\hline 19 & Michael J. Carey & 45.8 \\
\hline 20 & David J. DeWitt & 45.1 \\
\hline
\end{tabular}

\section{Individual Frequency}

The leading research contributors based on the research work in the Topic_Paper_Author dataset were ranked by using the IF method. Table 1.5 lists the top 20 leading contributors in the dataset. By using the Individual Frequency (IF) method, the leading author with the highest frequency (80.5) was identified as Mr. Jiawei Han. The other authors who formed the top nineteen ranking include Mr. Philip S. Yu (79.8), Mr. Surajit Chaudhuri (68.5), Mr. Hector Garcia-Molina (66.8), Mr. Rakesh Agrawal (65.3), Mr. Christos Faloutsos (68), Mr. H. V. Jagadish (64), Mr. Jeffrey F. Naughton (64), Mr. Divesh Srivastava (62), Mr. Michael J. Franklin (62), Mr. Jennifer Widom (61), Mr. Hans-Peter Kriegel (60), Mr. Rajeev Motwani (60), Mr. Richard T. Snodgrass (60), Mr. Arie E. Kaufman (58), Mr. S. Muthukrishnan (58), Mr. David J. DeWitt (55), Mr. Michael Stonebraker (55), Mr. Raghu Ramakrishnan (52), and Mr. Michael J. Carey (51).

\section{Weighted Frequency}

The leading research contributors based on the research work in the Topic_Paper_Author dataset were ranked by using the WF method. Table 1.6 lists the top 20 leading contributors in the dataset. By using the WF method, the leading author with the highest frequency (80.5) was identified as Mr. Jiawei Han. The other authors who formed the top nineteen ranking include Mr. Philip S. Yu (79.8), Mr. Surajit Chaudhuri (68.5), Mr. Hector Garcia-Molina (66.8), Mr. Rakesh Agrawal (65.3), Mr. H. V. Jagadish (42.68), Mr. Michael J. Franklin (38.11), Mr. Baruch Awerbuch (38), Mr. Philip S. Yu (37.61), Mr. Hector Garcia-Molina (36.81), Mr. Michael J. Carey (34.82), Mr. Michael Stonebraker (34.56), Mr. Christos Faloutsos (33.53), Mr. Jon M. Kleinberg (32.83), Mr. Piotr Indyk (31.95), Mr. Charu C. Aggarwal (31), Mr. Marianne Winslett (31), Mr. Pankaj K. Agarwal (30.83), Mr. SudiptoGuha (30.36), and Mr. Kenneth A. Ross (30.29). 
Table 1.5 The top 20 authors ranking by using Individual Frequency (IF) method

\begin{tabular}{|c|c|c|}
\hline S.No. & Author & Individual_Frequency \\
\hline 1 & Philip S. Yu & 100 \\
\hline 2 & Jiawei Han & 93 \\
\hline 3 & Hector Garcia-Molina & 78 \\
\hline 4 & Surajit Chaudhuri & 72 \\
\hline 5 & Rakesh Agrawal & 70 \\
\hline 6 & Christos Faloutsos & 68 \\
\hline 7 & H. V. Jagadish & 64 \\
\hline 8 & Jeffrey F. Naughton & 64 \\
\hline 9 & Divesh Srivastava & 62 \\
\hline 10 & Michael J. Franklin & 62 \\
\hline 11 & Jennifer Widom & 61 \\
\hline 12 & Hans-Peter Kriegel & 60 \\
\hline 13 & Rajeev Motwani & 60 \\
\hline 14 & Richard T. Snodgrass & 60 \\
\hline 15 & Arie E. Kaufman & 58 \\
\hline 16 & S. Muthukrishnan & 58 \\
\hline 17 & David J. DeWitt & 55 \\
\hline 18 & Michael Stonebraker & 55 \\
\hline 19 & Raghu Ramakrishnan & 52 \\
\hline 20 & Michael J. Carey & 51 \\
\hline
\end{tabular}


Table 1.6 The top 20 authors ranking by using Weighted Frequency (WF) method

\begin{tabular}{|c|c|c|}
\hline S.No & Author & Weighted_Frequency \\
\hline 1 & Surajit Chaudhuri & 57.58 \\
\hline 2 & Rakesh Agrawal & 52.33 \\
\hline 3 & Jiawei Han & 49.45 \\
\hline 4 & NogaAlon & 46.5 \\
\hline 5 & Richard T. Snodgrass & 45.16 \\
\hline 6 & H. V. Jagadish & 42.68 \\
\hline 7 & Michael J. Franklin & 38.11 \\
\hline 8 & Baruch Awerbuch & 38 \\
\hline 9 & Philip S. Yu & 37.61 \\
\hline 10 & Hector Garcia-Molina & 36.81 \\
\hline 11 & Michael J. Carey & 34.82 \\
\hline 12 & Michael Stonebraker & 34.56 \\
\hline 13 & Christos Faloutsos & 33.53 \\
\hline 14 & Jon M. Kleinberg & 32.83 \\
\hline 15 & Piotr Indyk & 31.95 \\
\hline 16 & Charu C. Aggarwal & 31 \\
\hline 17 & Marianne Winslett & 31 \\
\hline 18 & Pankaj K. Agarwal & 30.83 \\
\hline 19 & SudiptoGuha & 30.36 \\
\hline 20 & Kenneth A. Ross & 30.29 \\
\hline
\end{tabular}

Table 1.7 represents all the methods for identifying the frequency of an author contribution up to ten authors in the research articles and considerations of their names commonly occurrence in all the existing and proposed method.

Table 1.7 Comparison of the high frequency authors by using the existing and proposed methods

\begin{tabular}{|l|l|l|l|l|l|l|l|}
\hline S. No. & Author & SDC & EC & FLAE & PCI & IF & WF \\
\hline 1 & Surajit Chaudhuri & 58.5 & 12.24 & 49.92 & 68.5 & 72 & $\mathbf{5 7 . 5 8}$ \\
\hline 2 & Rakesh Agrawal & 55 & 17.5 & 48 & 65.3 & 70 & 52.33 \\
\hline 3 & Jiawei Han & 56.5 & 13.02 & 30.58 & 80.5 & $\mathbf{9 3}$ & 49.46 \\
\hline 4 & Michael J. Franklin & 41.5 & 12.4 & 29.5 & 55.5 & 62 & 38.12 \\
\hline 5 & Christos Faloutsos & 41 & 11.56 & 23.84 & 57.1 & 68 & 33.53 \\
\hline
\end{tabular}

Surajit Chaudhuri was having impact of frequencies respectively 58.5 in Sequence Determines Credit (SDC) method, 12.24 in Equal Contribution (EC) method, 49.92 in First Last Author Emphasis (FLAE) method, 68.5 in Percent Contribution Indicated (PCI) method, 72 in Individual Frequency (IF) method, and 57.58 in Weighted Frequency (WF) method.

Rakesh Agrawal was having impact of frequencies respectively 55 in Sequence Determines Credit (SDC) method, 17.5 in Equal Contribution (EC) method, 48 in First Last Author Emphasis (FLAE) method, 65.3 in Percent Contribution Indicated (PCI) method, 70 in Individual Frequency (IF) method, and 52.33 in Weighted Frequency (WF) method. 
Jiawei Han was having impact of frequencies respectively 56.5 in Sequence Determines Credit (SDC) method, 13.02 in Equal Contribution (EC) method, 30.58 in First Last Author Emphasis (FLAE) method, 80.5 in Percent Contribution Indicated (PCI) method, 93 in Individual Frequency (IF) method, and 49.46 in Weighted Frequency (WF) method.

Michael J. Franklin was having impact of frequencies respectively 41.5 in Sequence-Determines Credit (SDC) method, 12.4 in Equal Contribution (EC) method, 29.5 in First Last Author Emphasis (FLAE) method, 55.5 in Percent Contribution Indicated (PCI) method, 62 in Individual Frequency (IF) method, and 38.12 in Weighted Frequency (WF) method.

Christos Faloutsos was having impact of frequencies respectively 41 in Sequence Determines Credit (SDC) method, 11.56 in Equal Contribution (EC) method, 23.84 in First Last Author Emphasis (FLAE) method, 57.1 in Percent Contribution Indicated (PCI) method, 68 in Individual Frequency (IF) method, and 33.53 in Weighted Frequency (WF) method.

The existing one of the method Sequence Determines Credit (SDC) approach and our proposed framework Weighted Frequency (WF) methods provided almost the same result. However, in the case of papers with more than 10 authors, the results had a vast difference for both the methods.

The proposed systems, such as Individual Frequency (IF) and Weighted Frequency (WF), have no repeated impact for each position. However, all the existing systems have almost repeated impact for each of the researcher position.

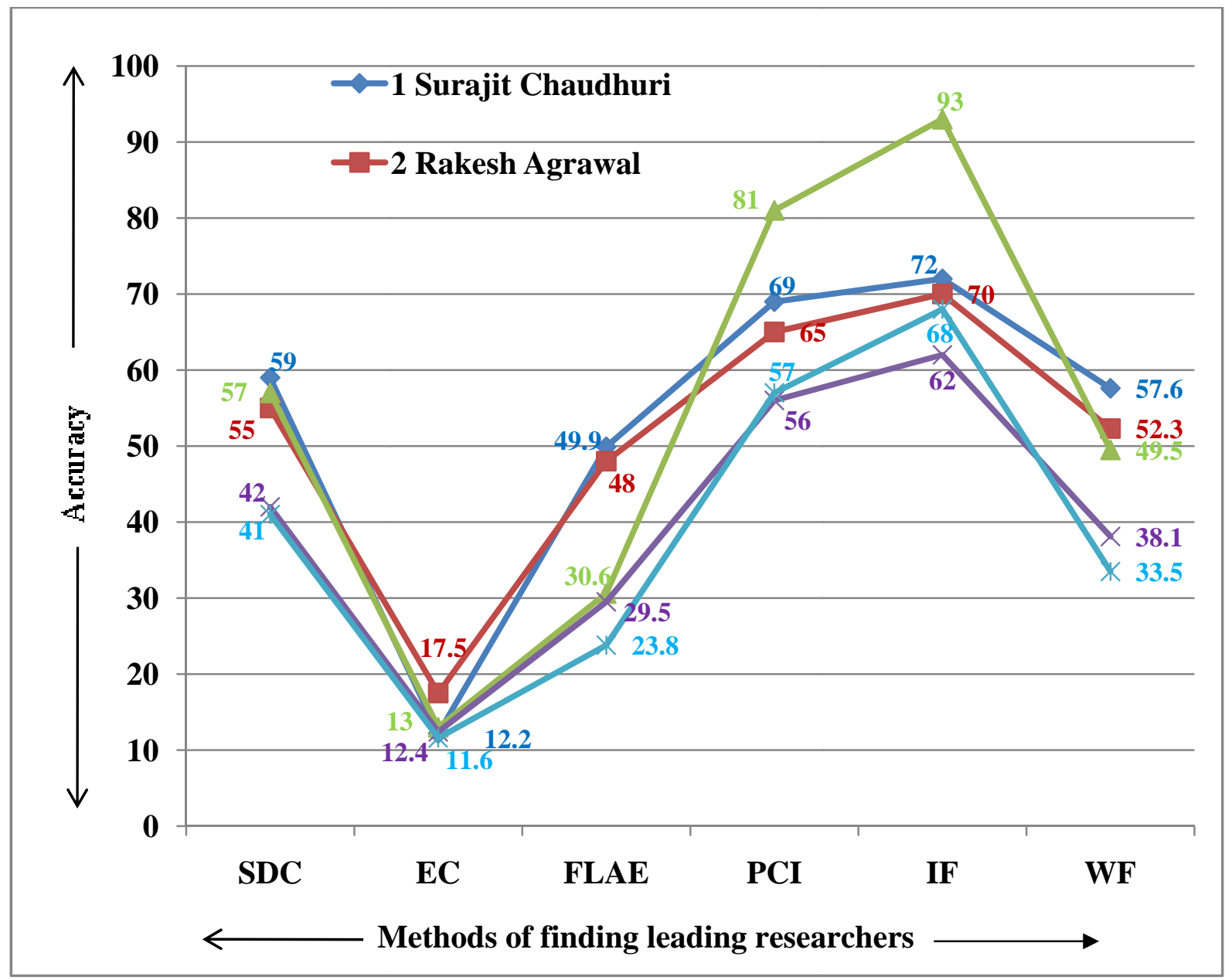

Figure 1.1 The representation of accuracies for the Proposed and Existing Systems.

The proposed framework strongly suggests the approaches for measuring the research contributions by authors in a research community. Although the Weighted Frequency method considers each position of the researcher, the Weighted Frequency (WF) calculation does not assign any repeated impact for more than one position for the researcher. The existing systems assigned the same weights for more than one positions. Therefore, the proposed system is more efficient and useful for the research society when compared with other existing systems. 


\section{CONCLUSION}

The proposed framework finds the leading research contributors based on the individual frequency and the weighted frequency. This system produces the better and reasonable accuracy compare than existing system. The proposed framework strongly suggests the approaches for measuring the research contributions by authors in a research community. Although the Weighted Frequency method considers each position of the researcher, the Weighted Frequency (WF) calculation does not assign any repeated impact for more than one position for the researcher. The existing systems assigned the same impact factor values for more than one positions. Therefore, the proposed system produces the efficient and reasonable accuracy for the research and academic society compare than other existing systems.

\section{References:}

[1] Jie Tang, Jing Zhang, Limin Yao, Juanzi Li, Li Zhang, and Zhong Su. ArnetMiner: Extraction and Mining of Academic Social Networks. In Proceedings of the Fourteenth ACM SIGKDD International Conference on Knowledge Discovery and Data Mining (SIGKDD'2008). pp.990-998.

[2] T. Tscharntke, M. E. Hochberg, T. A. Rand, V. H. Resh, and J. Krauss, "Author sequence and credit for contributions in multiauthored publications,” PLoS Biol., vol. 5, no. 1, pp. 0013-0014, 2007.

[3] http://www.cs.waikato.ac.nz/ml/weka/

[4] https://meshb.nlm.nih.gov/MeSHonDemand

[5] https://www.sas.com/en_us/software/university-edition/download-software.html

[6] https://support.sas.com/en/support-home.html

[7] https://www.ultraedit.com

[8] M. T. Rahman, J. Mac Regenstein, N. L. A. Kassim, and N. Haque, "The need to quantify authors' relative intellectual contributions in a multi-author paper,” J. Informetr., vol. 11, no. 1, pp. 275-281, 2017.

[9] Tasleem Arif, "Exploring the Use of Hybrid Similarity Measure for Author Name Disambiguation,” International Journal of Science and Technology Research, vol. 4, no. 12, pp. 171-175, 2015.

[10] J. M. Warrender, “A Simple Framework for Evaluating Authorial Contributions for Scientific Publications," Sci. Eng. Ethics, vol. 22, no. 5, pp. 1419-1430, 2016. 